\title{
Analysis of two- and three-particle motion in a Couette cell
}

\author{
M. Popova, P. Vorobieff \& M. Ingber \\ Department of Mechanical Engineering, \\ The University of New Mexico, Albuquerque, New Mexico, USA
}

\begin{abstract}
We present an experimental investigation of the irreversibility of two and three spherical particle interactions in shear flow. The experiment is performed in a stratified two-dimensional fluid inside a Couette cell at a very low Reynolds number. The particles are placed into the cell in well-characterized initial positions. Their motion is driven by the inner wall of the cell that is repeatedly rotated by the same angle clockwise and then counterclockwise. Nominally the flow is completely reversible (if the particles do not come close to each other, they return to their initial positions). Three types of particles are used with different surface roughnesses. In two-particle interactions, the degree of irreversibility on the macroscopic scale is found to be correlated with the average microscopic roughness of the particles. Subsequently, we investigate three-particle interaction and find an appreciably different behaviour, suggesting that forces in a multiparticle system cannot be reduced to force-pairs between individual particles.
\end{abstract}

\section{Introduction}

Addition of a suspended phase (gas bubbles, particles, or droplets) to a fluid flow appreciably complicates the challenge of predicting the flow, either theoretically or numerically. In many modern applications, especially in the emerging areas of micro- and nanoscale processing, the flow regime of most interest is viscous, nonlinear shear suspension flow. It may occur during composite and ceramic processing, production of semiconductors and magnetic storage media, and encapsulation of electronic components. Viscous shear flows with particle suspensions are also important in such earth, environmental, and planetary science problems as transport of sediments, contaminants, and slurries, and secondary oil recovery by hydraulic fracturing. 
The main challenge in addressing these applied problems lies in the necessity to relate between the physical phenomena on the scale of individual particles and the resulting macroscopic behaviour of the flow, and so far, development of rheological models predicting suspension flows has been only partially successful, despite major advancements in the field in the last two decades (see [1-5] and papers cited therein). One of the important macroscopic characteristics of suspension flows is the particle concentration profile. Existing models can predict such profiles well only for steady states, which are rarely achieved in many suspension flow applications, where the flows remain transient. A common example of such an application is fluid moving in the annular region between two rotating cylinders (Couette flow). For simplicity, many researchers consider the particles seeding the flow to be spheres of uniform size. For a constant concentration of such spheres, dimensional considerations used in rheological models lead to a prediction that the rate of particle migration in Couette flow should scale as the square of the sphere radius. However, experiments [6] reveal that the actual scaling is closer to the cube of that radius. Moreover, if the current models are used to predict the time required for a Couette flow to achieve steady state, the model prediction and the time measured in experiment can differ by orders of magnitude.

Arguably the main reason for this discrepancy is the highly non-conservative nature of the forces in multi-particle suspension systems, causing such systems to exhibit strongly irreversible behaviour. In contrast, the governing equations for the pure fluid flow in many viscous shear-flow systems are the Stokes equations, which are reversible in time. To reconcile the models with reality, the physical mechanisms of irreversibility must be understood and accounted for.

One of the proposed mechanisms of irreversibility is due to microscopic surface roughness, originally suggested by Arp and Mason [7]. Interaction between microscale features on particle surfaces causes the particles to "stick" together, thus producing irreversibility. The same kind of roughness effect would also make the process of a heavy particle settling towards the bottom of a fluid-filled container irreversible (in this case, due to the particle "sticking" to the bottom), as the experiments of Smart and Leighton [8] confirm. This study also found a qualitative agreement between the hydrodynamic roughness as the measure of irreversibility of the particle interaction with the container wall and the actual surface roughness of the particle according to profilometry measurements. In these two studies, roughness effects were assumed to be the sole source of irreversibility. A recent investigation [9] of two-particle interaction in Couette flow confirms this notion, establishing a quantitative correlation between irreversibility in the flow and average surface roughness of the particles as measured with a scanning electron microscope (SEM).

But can the interaction between multiple particles in a suspension be reduced to particle-pair interactions? It has been almost universally assumed in previous works that the most important interactions are those in particle pairs, with the force balance for a multi-particle system obtained as the sum of binary forces. This notion, however, was recently shown to be theoretically incorrect for a dense configuration of particles [10]. Thus another source of irreversibility 
due to nonlinear multi-particle interactions may exist in suspension flows. Its existence could account for the chaotic behaviour of the trajectories of perfectly smooth particles in numerical simulations of three-particle interactions in nominally reversible viscous flow $[11,12]$, where any surface roughness-related irreversibility is eliminated in the numerical formulation of the problem.

In this paper, we present an experimental study of two- and three-particle interactions in a viscous Couette cell flow attempting to elucidate the influence of the number of particles involved in the interaction on the irreversible behaviour manifested by the flow.

\section{Experimental arrangement}

Our experimental apparatus (Fig. 1) is a wide-gap Couette cell with the outer (stationary) cylinder diameter $D_{o}=20 \mathrm{~cm}$ and the inner (rotating) cylinder diameter $D_{i}=10 \mathrm{~cm}$. The outer cylinder is machined from acrylic polymer together with the stationary bottom plate, while the inner cylinder can be rotated by an electric stepper motor ("Compumotor," Fig. 1) via a belt drive. An arbitrary motion profile for the rotating cylinder can be downloaded to the computer interface controlling the motor ("Compumotor controller," Fig. 1). The cell is $4 \mathrm{~cm}$ deep and filled with a vertically stratified viscous fluid (water solution of $\mathrm{ZnCl}_{2}$ and commercial water-miscible solvent Triton X-100). The refraction index of the fluid (1.49) matches that of the outer cylinder of the cell, to facilitate imaging of the side views of the cell and minimize refraction off boundaries. The density and stratification of the fluid render the PMMA (polymethyl methacrylate) particles used in experiment neutrally buoyant at a depth of $1 \mathrm{~cm}$. The specific density of PMMA at this depth is 1.05 , and the overall density difference from top to bottom of the cell due to stable stratification is about lin fluid viscosity due to stratification. We conducted rheometry measurements to obtain the kinematic viscosity of the fluid $\nu=146 \mathrm{~cm}^{2} / \mathrm{s}$. The rheometry measurements also showed a linear relationship between torque and strain with no memory effects. Thus the fluid can be considered Newtonian (i.e., shear stress in it is linearly proportional to the velocity gradient).

The PMMA particles used in the experiments have been individually mapped using a scanning electron microscope. Thus for each individual particle, the rms average and the peak values of surface non-uniformities are known. During the experiments, two or three PMMA particles of nominal diameter $d_{p}=0.635 \mathrm{~cm}$ were placed into the cell using a template - a flat acrylic plate with holes positioned at the same location with respect to the outer cylinder, ensuring consistency of the initial positions of the particles in the pair or triplet. After the particles settled to their neutral buoyancy depth, the inner cylinder was rotated $750^{\circ}$ forward (clockwise if viewed from above the cell). Then the direction of the rotation was reversed, and the inner cylinder was rotated $750^{\circ}$ backward (clockwise) at the same angular velocity. The characteristic dimensionless parameter describing the 

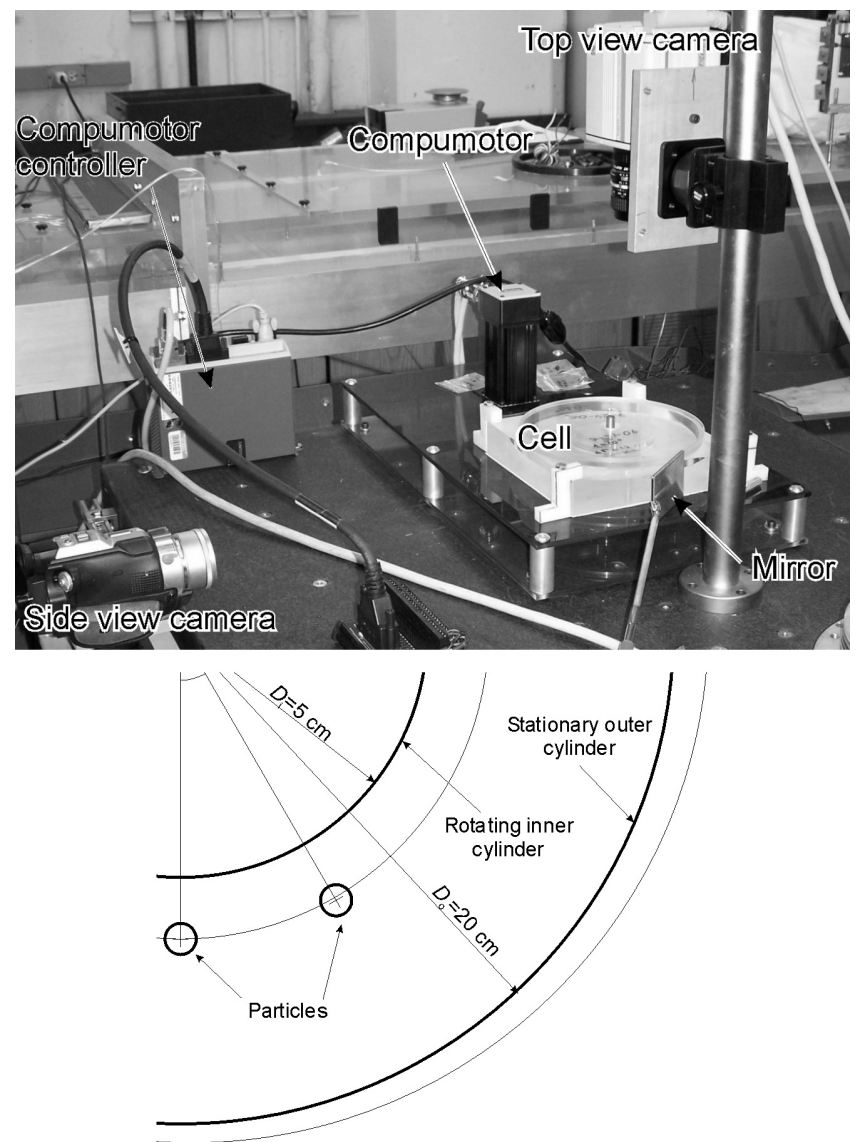

Figure 1: Experimental setup. Top: photograph of the actual arrangement with individual components labeled. Bottom: schematic of the partial view of the cell from above with dimensions.

relative importance of dissipation with respect to inertia in the flow is the largescale Reynolds number

$$
\frac{R e=\left(\pi f D_{i}\right)\left(D_{o}-D_{i}\right)}{\nu,}
$$

where $f$ is the rotation frequency. In the experiments described here, $R e \sim 0.01$, indicating that the flow is laminar and should be nominally consistent with the Stokes approximation $(R e<<1)$ for viscous time-reversible flow.

The experiment was visualized by a ten-bit grayscale digital camera with a pixel resolution of 1536 by 1024 . The camera was positioned directly above the Couette cell ("Top view camera," Fig. 1). The top view of the cell was augmented 
by side views captured with another camera through the flat side of the acrylic block and through mirrors at about $45^{\circ}$ to the adjacent sides. These side views we used to verify the two-dimensionality of the particle interactions. During the experiments, the image capture rate was maintained at $0.25 \mathrm{~Hz}$, thus yielding an image sequence of 72 captures per experimental run. The camera spatial resolution was $0.20 \mathrm{~mm} / \mathrm{pixel}$, thus the characteristic particle size was 31.2 pixels. Prior to each experimental run, an image of the experiment without particles was captured. In postprocessing, this image was subtracted from the image of the cell with particles, thus making it possible to enhance the image contrast. Then centroids of the particles were found using a standard algorithm [13] and converted to polar coordinates $r, \theta$ with the center corresponding to the center of rotation of the neutral buoyancy plane (i.e., the axis of rotation of the inner cylinder).

In the experiments described in the following section, the smoothest particles at our disposal were used. Their characteristic roughness is $250 \mathrm{~nm}$, or about $10^{-4}$ of the particle radius. In relative terms, this roughness is appreciably lower than that of the particles used in the experiments of Arp and Mason [7] and Smart and Leighton [8].

\section{Observations and analysis}

Here we consider the Couette flow with either two or three particles in the fluid. The initial conditions produced by the template as described in the previous section have the particles nominally separated by $10^{\circ}$ in the tangential $(\theta)$ direction. The differences in the radial distances are nominally 0.025 of the inner cylinder radius $r_{i}$ between two particles in a pair. For the case of three particles, the initial radial distance increases by the same amount $\left(0.025 r_{i}\right)$ between each particle. The actual initial conditions may differ by some small amount from the nominal initial conditions set by the template. These deviations develop during the settling of the particles to the neutral buoyancy depth. The velocity imparted by the rotation of the Couette cell to the particle closest to the inner cylinder is the highest, so it "catches up" to the next particle. This results in what we refer to as "particle interaction," with the particles in close proximity to each other and moving as a pair. The resolution of our acquisition system is not sufficient to tell whether the micro-roughness features on the surface of the particles are actually in contact, thus we chose to use the term "interaction" to leave a certain ambiguity.

Figure 2 shows a superposition of images of a two-particle experiment, with the image on the left corresponding to the forward (clockwise) rotation of the inner cylinder and the image on the right showing the reverse (counterclockwise) part of the cycle. Particle pairs in each sequence are labeled using the angle $\phi$ of the rotation of the inner cylinder, $\phi=0$ corresponding to the initial and the final conditions. The "trailing" particle in the pair, initially positioned closer to the rotating cylinder, is marked with a white dot. In the second (counterclockwise rotation) picture, initial positions of the two particles are marked with contours.

From the image sequence, it is clear that the close interaction of the two particles brings irreversibility to the flow. The particles do not return to the initial positions. 

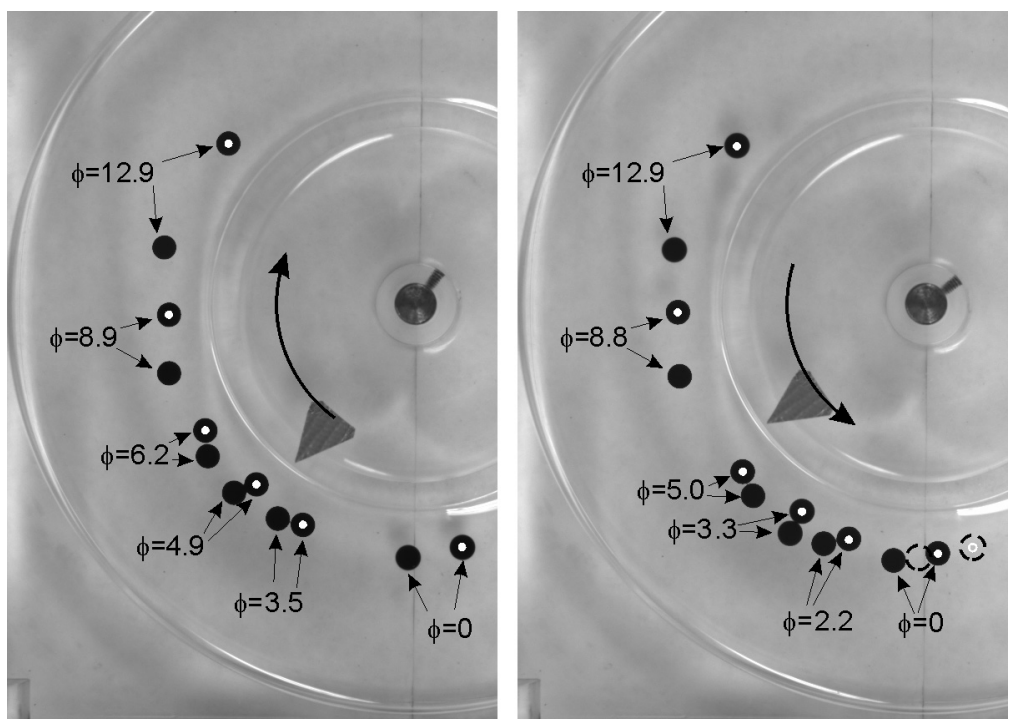

Figure 2: Forward (left) and reverse (right) parts of an experimental run with two particles. One of the particles is marked with a white dot for ease of identification (the dot is not physically present during the experiments). Values of the angle of rotation of the inner cylinder $\phi$ are shown for each particle pair. Dashed contours in the right image show the initial positions of the particles.

Moreover, the interactions themselves take place at different ranges of $\phi$ for the forward and reverse parts of the cycle: $3.5<\phi<6.2$ during clockwise rotation of the inner cylinder and $5.0>\phi>2.2$ during the counterclockwise rotation. This property of the interaction becomes even more apparent if the trajectories of the particles are plotted in the plane of polar coordinates $(r, \theta)$. Figure 3 shows such trajectories for two realizations of the experiment with different initial conditions, with the plot on the left corresponding to the image sequence of Fig. 2.

Despite the differences in the initial conditions, both experimental realizations manifest themselves similarly in the $(r, \theta)$ plane. It is also apparent from the trajectories in Fig. 3 that the center of gravity of the particle pair moves away from the rotating cylinder, in good agreement with earlier experimental results [9]. While the particles do not return to their initial positions, their respective positions are retained (the "trailing" particle marked by the white dot in Fig. 2 returns to its "trailing" place counterclockwise of the unmarked particle).

How does the addition of a third particle change the behaviour of the system? Figure 4 shows a characteristic image sequence constructed in the same way as the sequence of Fig. 2, but with artificial markers now identifying the "middle" and the "trailing" particle, and the distance from the inner cylinder of the Couette cell 


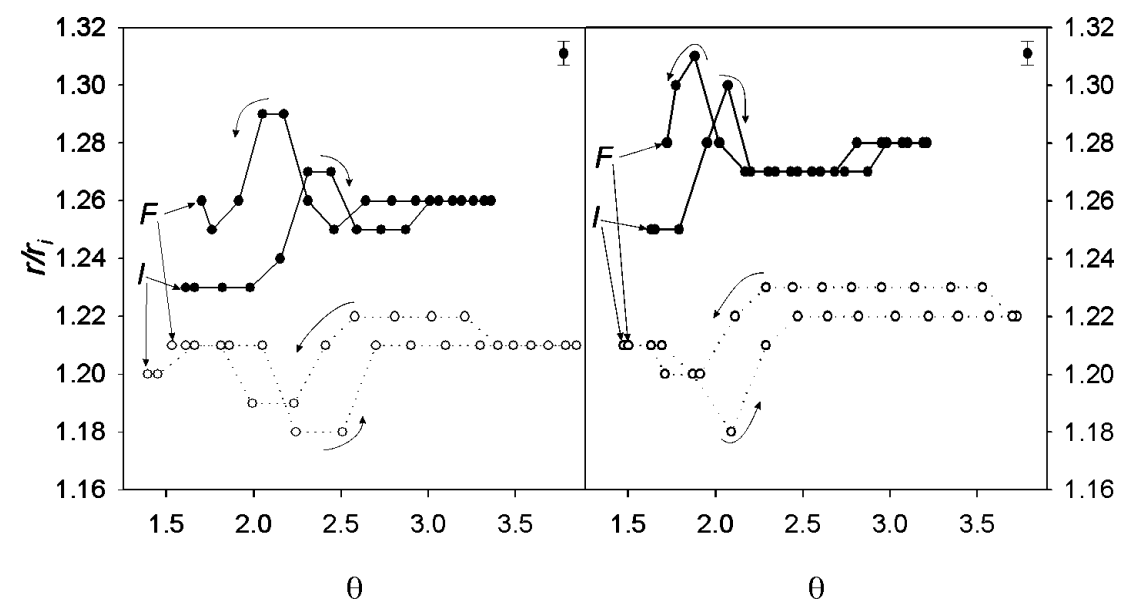

Figure 3: Polar coordinates $(r, \theta)$ of two particles during the forward-reverse cycle of the inner cylinder rotation. Plot on the left corresponds to Fig. 2, plot on the right was obtained from different initial conditions (greater initial distance from the inner cylinder). Representative error bars are in the upper right corner of each plot. Letters "I" and "F" denote the initial and final positions of the particles. Radius $r$ is normalized by the inner cylinder radius $r_{i}$, angle $\theta$ is in radians, measured in the clockwise direction from a horizontal axis passing through the center of rotation.

increasing in the initial conditions from the "trailing" to the "middle" and to the "leading" (unmarked) particle.

There are several appreciable differences between the two- and three-particle cases. First, while the particles in a pair have a close interaction once during the forward and reverse parts of the cycle, particles in a triplet may have a different number of such encounters. In Fig. 4, the forward part of the cycle shows one such interaction beginning at $\phi=8.3$. This interaction causes the "middle" and the "leading" particles to exchange their order with respect to the direction of rotation. A similar exchange takes place in Fig. 2. However, during the reverse part of the three-particle experiment, two close approaches happen. The first one, occurring around $\phi=8.8$, involves the "middle" and the "leading" particle again, with their initial order restoring itself. Then another close encounter happens, this time involving the "middle" and the "trailing" particle around $\phi=3.9$. As the result, these two particles reverse their order in the $\theta$ coordinate direction. The initial order of the particles was "leading," "middle," "trailing." In Fig. 4, the final order of the particles is "leading," "trailing," "middle." Without detailed knowledge of the particle trajectories, it would not be possible to tell which one is which! This behaviour bears an uncanny resemblance to the chaotic "dance" of three sedimenting particles in the original experiments of Jayaweera et al.[14] 

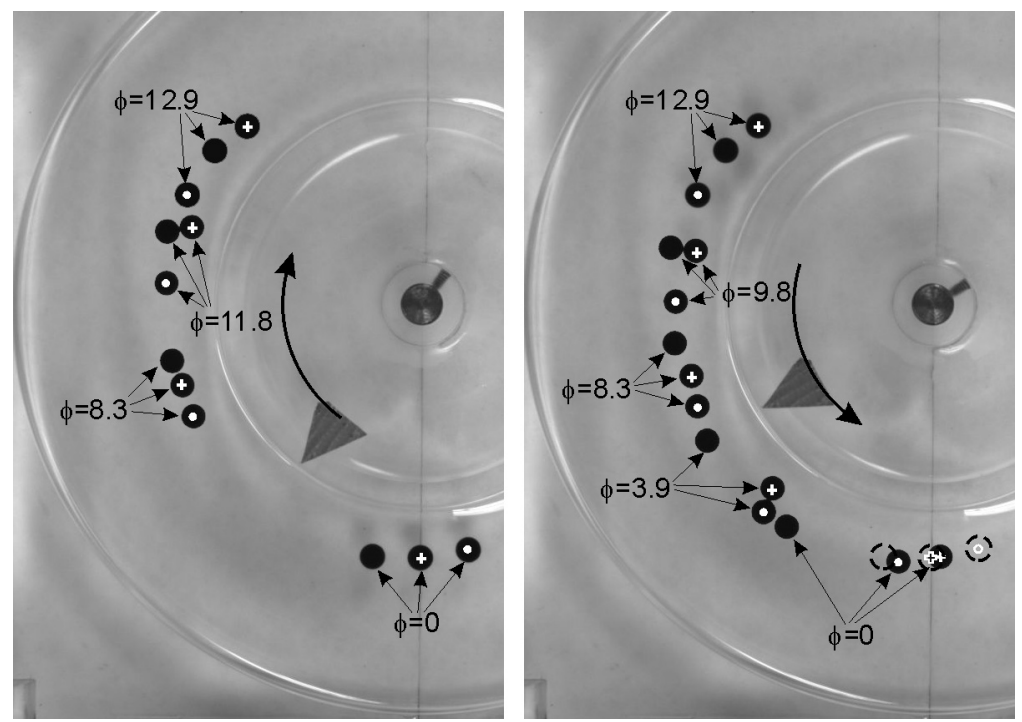

Figure 4: Forward (left) and reverse (right) parts of an experimental run with three particles. One particle (initially closest to the rotating cylinder) is marked with a white dot for ease of identification. The particle in the middle is marked with a "+" sign. The particle initially farthest from the rotating cylinder is unmarked. The marks are not physically present during the experiments. Values of the angle of rotation of the inner cylinder $\phi$ are shown for each particle triplet. Dashed contours in the right image show the initial positions of the particles.

and the numerical experiments of Jánosi et al. [12]. For additional elucidation of these features, Fig. 5 shows the particle trajectories in the $(r, \theta)$ plane for the experimental run depicted in Fig. 4.

\section{Conclusion}

Our experimental study shows that the behaviour of a system of three particles in viscous, stably stratified radial shear flow is appreciably different from the behaviour of a particle pair under similar conditions. The behaviour of the particle pair is irreversible in the sense that the particles do not return to their initial condition upon reversal of the flow. This irreversibility is also present in the threeparticle case. There also exists a shared trend for the "center of mass" of the particle system to move away from the inner, rotating cylinder of the Couette cell towards the lower shear rate region of the flow field. However, the threeparticle system manifests a greater degree of unpredictability in the sense that, without the knowledge of the particle trajectories, it is impossible to tell which 


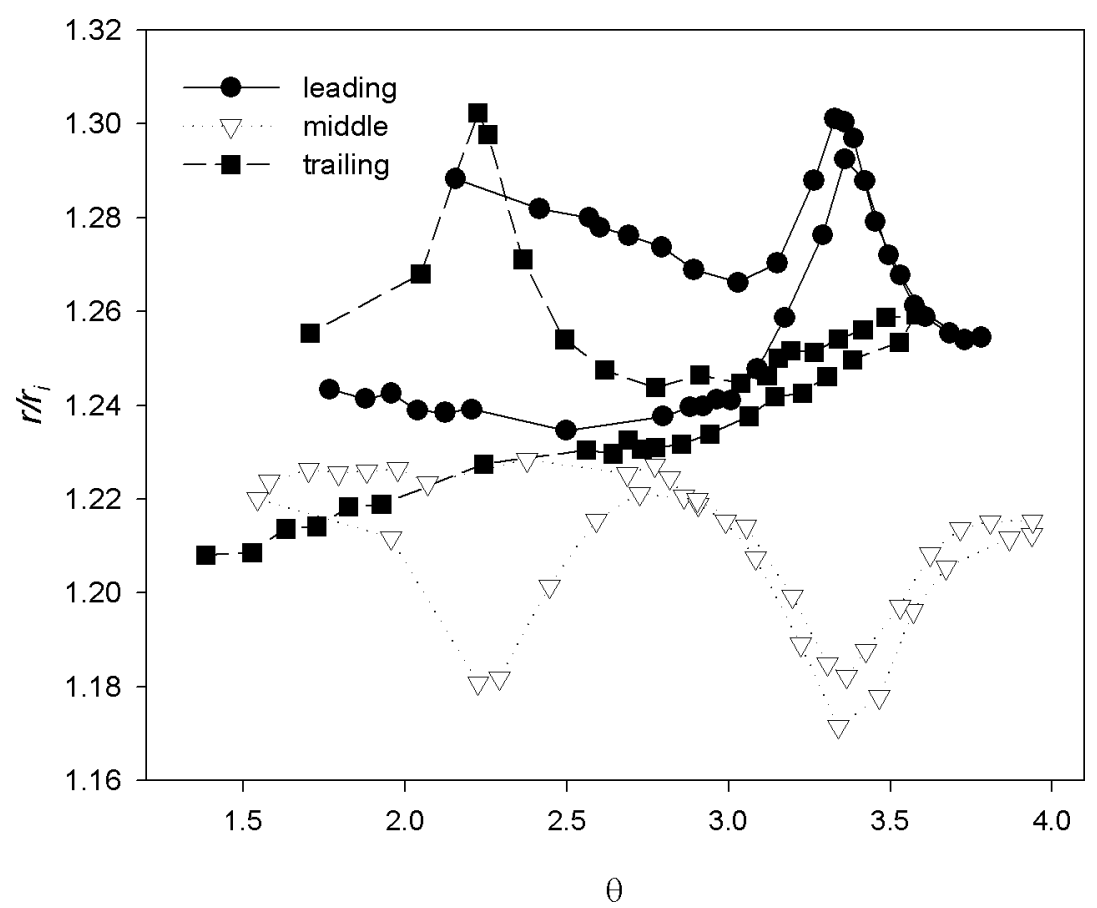

Figure 5: Polar coordinates $(r, \theta)$ of three particles during the forward-reverse cycle of the inner cylinder rotation. This plot corresponds to Fig. 4. Refer to the text for the description of the initial positions of the leading, middle, and trailing particle. Radius $r$ is normalized by the inner cylinder radius $r_{i}$, angle $\theta$ is in radians, measured in the clockwise direction from a horizontal axis passing through the center of rotation.

particle corresponds to which when comparing their initial and final positions. The presence of a third particle greatly increases the system sensitivity to small fluctuations in the initial conditions. These behaviours are observed for fairly smooth (relative roughness $\sim 10^{-4}$ ) spheres.

\section{Acknowledgement}

This work was partially supported by the US Department of Energy (DOE) grant DE-FG02-05ER25705. The financial support does not constitute an endorsement by the DOE of the views expressed in this paper. 


\section{References}

[1] Phillips, R., Armstrong, R., Brown, R., Graham, A., \& Abbott, J., Numerical analysis of normal stress in non-Newtonian boundary layer flow. Physics of Fluids A, 4(1), pp. 30-40, 1992.

[2] Buyevich, I., Particle distribution in suspension shear flow. Chemical Engineering Science, 51(4), pp. 635-647, 1995.

[3] Morris, J. \& Brady, J.F., Pressure-driven flow of a suspension: Buoyancy effect. International Journal of Multiphase Flow, 24(1), pp. 105-130, 1998.

[4] Fang, Z., Mammoli, A., Brady, J., Ingber, M., Mondy, L. \& Graham, A., Pressure-driven flow of a suspension: Buoyancy effect. International Journal of Multiphase Flow, 28(1), pp. 137-166, 2002.

[5] Pozarnik, M. \& Skerget, L., Boundary element method numerical model based on mixture theory of two-phase flow. Computational Methods in Multiphase Flow II, eds. A. Mammoli \& C. Brebbia, WIT Press: Southampton, UK, pp. 3-12, 2003.

[6] Tetlow, N., Graham, A., Ingber, M., Subia, S., Mondy, L. \& Altobelli, S., Particle migration in a couette apparatus: Experiment and modeling. Journal of Rheology, 42(2), pp. 307-327, 1998.

[7] Arp, P. \& Mason, S., Kinetics of flowing dispersions. 9. Doublets of rigid spheres (Experimental). Journal of Colloid and Interface Science, 61(1), pp. 44-61, 1977.

[8] Smart, J. \& Leighton, D., Measurement of the hydrodynamic surfaceroughness of noncolloidal spheres. Physics of Fluids A - Fluid Dynamics, 1(1), pp. 52-60, 1989.

[9] Popova, M., Vorobieff, P., Ingber, M. \& Graham, A., Interaction of two particles in a shear flow. Physical Review E, 2007. Submitted for publication.

[10] Putkaradze, V., Holm, D. \& Weidman, P., 2007. Preprint.

[11] Wang, Y., Mauri, R. \& Acrivos, A., The transverse shear-induced liquid and particle tracer diffusivities of a dilute suspension of spheres undergoing a simple shear flow. Journal of Fluid Mechanics, 327, pp. 255-272, 19996.

[12] Janosi, I., Tel, T., Wolf, D. \& Gallas, J., Chaotic particle dynamics in viscous flows: The three-particle Stokeslet problem. Physical Review E, 56(3), pp. 2858-2868, 1997.

[13] Prasad, A., Adrian, R., Landreth, C. \& Offutt, P., Effect of resolution on the speed and accuracy of particle image velocimetry interrogation. Experiments in Fluids, 13(2-3), pp. 105-116, 1992.

[14] Jayaweera, K., Mason, B. \& Slack, G., The behaviour of clusters of spheres falling through a viscous fluid. Part 1. Experiment. Journal of Fluid Mechanics, 20(1), pp. 121-128, 1964. 